\title{
Philosophiques
}

\section{Géométrie, fiction et discours sous hypothèse : Husserl et les objets intentionnels en 1894}

\section{Guillaume Fréchette}

Volume 36, numéro 2, automne 2009

Edmund Husserl (1859-1938)

URI : https://id.erudit.org/iderudit/039476ar

DOI : https://doi.org/10.7202/039476ar

Aller au sommaire du numéro

\section{Éditeur(s)}

Société de philosophie du Québec

ISSN

0316-2923 (imprimé)

1492-1391 (numérique)

Découvrir la revue

Citer cet article

Fréchette, G. (2009). Géométrie, fiction et discours sous hypothèse : Husserl et les objets intentionnels en 1894. Philosophiques, 36(2), 355-379.

https://doi.org/10.7202/039476ar
Résumé de l'article

Dans l'essai Objets intentionnels de 1894, Husserl développe en réaction à Twardowski une théorie originale de l'assomption comme solution au problème des représentations sans objet. Après avoir examiné le détail de cette théorie et en avoir soulevé les difficultés, je montre dans cet article que la solution proposée par cette théorie doit être abordée de manière indépendante de celle qui sera développée plus tard dans les Recherches logiques et j'expose dans quelle mesure elle est ancrée dans la psychologie descriptive brentanienne tout en mettant à profit certains outils de la logique de Bolzano. Enfin, j'indique que Husserl continuera à développer cette théorie après les Recherches logiques, confirmant ainsi qu'elle occupe une place de choix dans la théorie phénoménologique du jugement. 


\title{
Géométrie, fiction et discours sous hypothèse: Husserl et les objets intentionnels en 1894
}

\author{
GUILLAUME FRÉCHETTE \\ Université du Québec à Montréal
}

\begin{abstract}
RÉSUMÉ. - Dans l'essai Objets intentionnels de 1894, Husserl développe en réaction à Twardowski une théorie originale de l'assomption comme solution au problème des représentations sans objet. Après avoir examiné le détail de cette théorie et en avoir soulevé les difficultés, je montre dans cet article que la solution proposée par cette théorie doit être abordée de manière indépendante de celle qui sera développée plus tard dans les Recherches logiques et j'expose dans quelle mesure elle est ancrée dans la psychologie descriptive brentanienne tout en mettant à profit certains outils de la logique de Bolzano. Enfin, j'indique que Husserl continuera à développer cette théorie après les Recherches logiques, confirmant ainsi qu'elle occupe une place de choix dans la théorie phénoménologique du jugement.
\end{abstract}

\begin{abstract}
In his essay Intentional Objects of 1894, Husserl develops in response to Twardowski an original theory of assumptions as a solution to the problem of objectless presentations. First, I analyze in this paper the main points of his theory and point out some of the difficulties it raises. I then suggest that the solution presented in this theory must be addressed independently of the one developed by Husserl later in the Logical Investigations and try to show in which extent his theory of assumptions is rooted in the brentanian descriptive psychology, although it makes good use of some logical tools from Bolzano's Wissenschaftslehre. Since Husserl continues to work on this theory even after the Logical Investigations, it confirms the important place one should give to the theory of assumptions it the phenomenological theory of judgment.
\end{abstract}

\section{Remarques préliminaires. La place d'Objets intentionnels dans le corpus husserlien}

C'est en 1894, avec la parution à Vienne de la thèse d'habilitation de Twardowski sur le contenu et l'objet des représentations, que le problème des représentations sans objet réapparaît au menu des débats philosophiques dans l'Empire austro-hongrois. Mais la petite histoire de ce problème avait en fait déjà débuté une soixantaine d'années plus tôt, dans la métropole bohème de l'Empire, sous la plume de Bolzano. Au $\mathbb{S} 67$ de sa Wissenschaftslehre, Bolzano défend la thèse selon laquelle il y a des représentations auxquelles ne correspond aucun objet. Ainsi, ma représentation d'une montagne d'or ou d'un carré rond - les épisodes mentaux individuels - ont certes une matière (Stoff), mais elles n'ont pas d'objet. La matière de ces représentations est ce que Bolzano appelle la représentation objective d'une montagne d'or ou d'un carré rond. Selon Bolzano, ces représentations sont sans objet parce que la proposition qu'il y a un objet possédant de telles 
propriétés contredit, dans le premier cas, une vérité empirique, et dans le deuxième cas, une vérité conceptuelle.

Prenant pour point de départ les thèses de Brentano sur l'intentionnalité et l'objectualité des phénomènes psychiques, Twardowski tente de renverser les arguments de Bolzano en 1894. Avec un succès discutable. Car la position de Twardowski ne parvient pas à distinguer entre le caractère psychique des contenus de représentation et la signification identique exprimée dans différents actes. Malgré les difficultés inhérentes à l'entreprise de Twardowski, sa thèse d'habilitation de 1894 a soulevé une question qui, pour un philosophe comme Husserl reconnaissant tout autant l'influence de son maître Brentano que celle du logicien bohème Bolzano, n'est pas restée longtemps sans réponse. Comment peut-on défendre dans le cadre d'une seule théorie la thèse selon laquelle tout phénomène psychique est caractérisé par sa relation à un objet (ce qu'on appelle la thèse de l'intentionnalité de Brentano), et celle selon laquelle il y a des représentations qui n'ont pas d'objets (la thèse de Bolzano) ? Cette question est au cœur de celles qui ont le plus préoccupé Husserl entre 1893 et la publication des Recherches logiques en 1900-1901, et on peut dire que la réponse à celle-ci a été déterminante dans l'orientation prise par la phénoménologie dans cet ouvrage.

Un des témoignages les plus éloquents quant à l'effervescence de cette période dans le développement de la phénoménologie de Husserl est à trouver dans une lettre que Husserl adressa à Meinong le 5 avril 1902, dans laquelle il s'efforce à se dissocier de ce dernier sur à peu près tous les points que celui-ci soulève dans Über Annahmen comme étant des convictions communes aux deux auteurs. À cette lettre, il joint deux manuscrits d'une période antérieure aux Recherches logiques afin d'attester que, plusieurs années avant la publication d'Über Annahmen, il s'était déjà consacré au problème des hypothèses et des assomptions en théorie du jugement. Le premier de ces deux manuscrits a été écrit en réaction à Twardowski, et on le connaît aujourd'hui sous le titre d'Objets intentionnels. C'est ce texte de 1894 qui retiendra ici principalement notre attention ${ }^{1}$. Que Husserl ait considéré ce texte parmi ce qu'il avait de mieux à offrir après la publication des Recherches logiques, cela se confirme également par l'envoi du manuscrit à Daubert en 1904. Grâce à Daubert, le manuscrit circula alors à Munich, et on voit même Reinach y faire indirectement référence dans un article paru quelques années plus tard ${ }^{2}$.

1. Intentionale Gegenstände, abrégé ici par IG. La première version de ce texte était composée d'une première partie de 34 pages sur la distinction entre représentations subjectives et représentations objectives, qui est considérée aujourd'hui comme perdue. Cf. Schuhmann (1990), p. 137sq. sur cet aspect de l'histoire d'IG. Husserl avait rédigé en 1894 un texte prêt à être publié, intitulé Vorstellung und Gegenstand, qui comprenait la partie disparue ainsi que le texte aujourd'hui intitulé Intentionale Gegenstände, comme le rapporte Schuhmann (ibid., p. 141sq.)

2. Reinach (1911), p. 226sq. discute l'exemple du lion qu'on retrouve à plusieurs endroits dans $I G$. Schuhmann (2004), p. 126, a déjà noté cette référence implicite. Le rôle 
Mais ce qui fait surtout l'importance de l'essai Objets intentionnels dans le corpus husserlien - et c'est là le propos principal du présent exposé — tient à son introduction du concept d'assomption comme solution préliminaire au problème des représentations sans objet, solution qui dès lors, par extension, sera déterminante dans le développement du concept d'intentionnalité des Recherches logiques ${ }^{3}$. Si la plupart des interprètes de Husserl ont reconnu l'importance de ce texte dans le cheminement qui amène Husserl des thèses de la psychologie descriptive brentanienne vers la logique pure, il ne semble pas y avoir de consensus quant à l'interprétation à lui donner, ce qui a pour conséquence qu'Objets intentionnels est parfois employé pour défendre les thèses les plus variées quant aux origines et aux développements de la phénoménologie, allant jusqu'à une interprétation de l'influence de Twardowski sur Husserl quant au rôle de l'objet intentionnel dans la phénoménologie transcendantale ${ }^{4}$. Selon cette dernière interprétation, l'essai de 1894 ainsi que la «réaction à Twardowski» qu'il exprime seraient en fait un élément déclencheur tant de la critique du psychologisme qui sera développée dans les Prolégomènes que de la réduction phénoménologique développée dans les Idées directrices ${ }^{5}$. En fait, rien de tangible ne permet d'attester cette interprétation à la lumière des propos de Husserl lui-même sur ce texte ${ }^{6}$, et si l'enjeu n'est pas en soi dénué d'intérêt, il demeure accessoire par

central du concept d'assomption dans le développement de la phénoménologie de Husserl au milieu des années 1890 est à nouveau attesté grâce à la parution récente d'un texte contemporain d'Objets intentionnels, à savoir le manuscrit de 1893 Essai sur l'origine des concepts de nécessité et de conséquence nécessaire, sur le jugement hypothétique et le jugement causal, qui était le second des deux manuscrits envoyés par Husserl à Meinong. C $f$. "Versuch über den Ursprung der Begriffe "Notwendigkeit" und "notwendige Folge", über hypothetisches und kausales Urteil ", publié pour la première fois dans Hua XL, p. 1-30.

3. Parmi les premiers, Pietersma $(1986,315)$ a tôt vu l'importance du concept d'assomption dans le texte de 1894, et voit même une lacune dans le traitement que lui réserve Husserl dans la cinquième des Recherches logiques. Cependant, Pietersma identifie l'assomption telle qu'elle est discutée dans le texte de 1894 avec les caractères de position du $\$ 38$ de la cinquième des Recherches logiques, et comme je le montrerai dans la dernière section de ce texte, je crois qu'il se trompe sur ce dernier point.

4. Dans cet éventail d'interprétations, certains voient même dans le texte de 1894 une défense de la thèse qu'il n'y a pas de représentations sans objet et que Husserl y affirmerait que toute représentation a un objet intentionnel. Cf. Sepp (1988, p. 161).

5. Cf. Cavallin (1997 [1990], p. 28), English (1993), p. 16.

6. Que Husserl ait vu dans Objets intentionnels un texte "sur les assomptions ", c'est ce qu'attestent plusieurs de ses lettres, notamment celle à Meinong datée du 5 avril 1902 (Hua $B W 1$, p. 144) et celle à Daubert datée du 17 novembre 1904 (Hua BW2, p. 42), ainsi que les notes prises dans son journal personnel en 1906 ( $c f$. tout particulièrement Hua XXIV, 443). HuaXXIV, p. 443: "[D]ans cet ouvrage [=Über Annahmen], je ne trouve qu'une seule idée significative que je n'ai pas formulée dans mes Recherches logiques, bien que j'eusse déjà cette idée et que je l'avais considérée pendant l'élaboration, mais qu'en fin de compte, je n'osai pas incorporer [dans l'ouvrage]: la transposition des modifications de jugements en "simples représentations" au niveau des désirs et au niveau de tous les autres actes. Je possède encore le feuillet daté sur cette question (1894), dans lequel j'avais pris exactement la position de Meinong. Mais 
rapport à l'anti-psychologisme des Prolégomènes annoncé dans Objets intentionnels, par exemple.

Plus proches de la thèse que je propose ici, d'autres envisagent le texte de 1894 dans la lignée de Philosophie de l'arithmétique et y voient un exemple de la théorie "préphénoménologique» de l'intentionnalité. C'est le cas notamment de Fisette (2003, p. 64sq), qui attribue à l'échange avec Bolzano et Twardowski un rôle relativement instrumental, car le cœur du problème selon lui - le problème des représentations sans objet - est un problème auquel est confronté Husserl depuis plus longtemps et qui touche également le traitement des nombres imaginaires. Bolzano et Twardowski ne seraient ici que des interlocuteurs de circonstance dans une discussion déjà entamée depuis longtemps. Autrement dit, selon Fisette, la solution au problème des représentations sans objet dans le texte de 1894 serait partie intégrante de la solution au problème de la justification du calcul avec les nombres imaginaires que Husserl développe entre la parution de Philosophie de l'arithmétique (1891) et sa conférence de Göttingen de 1901 sur l'imaginaire en mathématiques ${ }^{7}$. Je ne suis pas en désaccord avec cette interprétation, mais je crois qu'il est plus profitable de garder les problèmes mathématiques relativement distincts des problèmes de psychologie descriptive et de logique dont traite Husserl dans cette période, car, comme je le montrerai, voir le texte de 1894 comme une solution unitaire à ce groupe de problèmes entraîne des difficultés qui peuvent être évitées autrement.

D'autres lectures d'Objets intentionnels ont été proposées depuis sa parution posthume en 1979, mais elles ne sont pas sans poser problème: Philipse (1987), par exemple, soutient que le texte de 1894 défend une conception non relationnelle de l'intentionnalité (en raison de sa généralisation du problème des représentations sans objet à la sphère de tous les actes mentaux) et que le plus grand mérite de ce texte repose dans la partie toujours portée disparue (!), dans laquelle Husserl développait, selon Philipse, une théorie de la signification ${ }^{8}$. Bref, la solution apportée par Husserl au problème des représentations sans objet l'aurait forcé à abandonner une conception relationnelle (brentanienne) de l'intentionnalité au profit d'une

j'ai vu alors de grandes difficultés, que Meinong ne voit pas, et elles m'empêchèrent de poursuivre." ("Ich finde in dem Buch [=Über Annahmen] nur einen bedeutsamen Gedanken, den ich in meinen Logischen Untersuchungen nicht ausgesprochen habe, obschon ich ibn während der Ausarbeitung hatte und erwog, aber dann doch nicht aufzunehmen wagte: die Übertragung der Modifikation von Urteilen in @bloßen Vorstellungen" auf Wünsche und alle sonstigen Akte. Ich besitze noch das datierte Blatt über diese Frage (1894), in dem ich genau Meinongs Position einnahm. Aber freilich, ich sah große Schwierigkeiten, die Meinong nicht sieht, und die hinderten mich, Folge zu geben»).

7. Reproduite dans Schuhmann \& Schuhmann (2001), p. 87sq.

8. Cf. particulièrement Philipse (1987), p. 311. Comme Pietersma, Rollinger (1996, p. 96 sq.; 1999, p. 149sq.) a bien vu la place centrale accordée à l'assomption dans Objets intentionnels et remarque à juste titre que le texte de 1894 n'est pas une simple "première version" des Recherches logiques, comme tente de le faire valoir Philipse. 
conception sémantique. En ce sens, suivant l'idée de Philipse, la clé du texte de 1894 serait en fait la première des $R L$ : une conception sémantique et non relationnelle de l'intentionnalité9. Je ne suis d'accord avec aucun des deux points avancés par Philipse: d'abord, il n'y a pas d'incompatibilité de principe entre la solution de Husserl au problème des représentations sans objet en 1894 et la conception "relationnelle ${ }^{10}$ de l'intentionnalité, comme on le verra dans notre discussion du concept d'assomption: au contraire, la théorie de l'assomption est conçue comme une solution au problème des représentations sans objet dans le contexte d'une conception brentanienne de l'intentionnalité ${ }^{11}$. L'autre point, selon lequel la solution du problème des représentations sans objet par Husserl en 1894 serait ultimement basée sur sa conception sémantique, est tout aussi discutable. Comme nous le verrons, la solution au problème des représentations sans objet présentée en 1894 jouit d'une relative indépendance face à celle avancée dans les Recherches logiques ${ }^{12}$.

\section{Husserl et le discours ex hypothesi dans Objets intentionnels}

Commençons par retracer les caractéristiques principales de la conception de l'assomption avancée par Husserl dans le texte de 1894. Husserl y ouvre la discussion du problème en opposant les deux thèses suivantes:
(A) À toute représentation ne correspond pas toujours un objet.
(B) Toute représentation représente un objet (C).

9. Ibid. De manière analogue à Philipse, Smith (1994, p. 130) interprète la stratégie d'Objets intentionnels exactement dans les termes de celle des Recherches logiques: "[T]hat the god Jupiter is an intentional object of my act is not to say that there is something, namely Jupiter, which lacks existence but is thought about by me. It is rather simply to say that my act is structured, qualitatively, in a certain way so that it is (a) describable as a presentation-of-thegod-Jupiter, and (b) such as to lack existence-presuppositions. » Cf. LU II/1, p. 399. L'interprétation de Münch (1993, p. 180sq.) va également dans cette direction. À l'extrême de cette tendance à assimiler la position d'Objets intentionnels à celle des Recherches logiques, tout en remettant en question la qualité des arguments de Husserl contre Twardowski, certains vont jusqu'à ignorer la théorie des assomptions de Husserl. C'est le cas récemment de Naberhaus (2006, p. 57sq.) et de Hickerson (2007, p. 107 et 114sq.).

10. Par «relationnel », Philipse veut désigner la conception classique de l'intentionnalité selon Brentano.

11. Du fait que Husserl dit que l'identité a lieu entre de "vrais » objets, et non des objets représentés, ou des objets immanents, Philipse lui fait défendre la thèse qu'il ne peut y avoir de relation sans que les relata existent, ce qui impliquerait du coup, selon lui, l'impossibilité pour Husserl de défendre une conception relationelle de l'intentionnalité.

12. Pour clore ce survol de la réception d'Objets intentionnels, mentionnons enfin l'adaptation originale par Künne (1995) de la théorie husserlienne des assomptions de 1894, qui propose une solution aux difficultés auxquelles fait face la sémantique frégéenne lorsqu'elle traite d'énoncés fictionnels. 
C'est à partir de l'opposition de ces deux thèses qu'émerge le soi-disant paradoxe des représentations sans objet que Husserl tente alors de résoudre. Dans ce qui suit, je vais exposer le concept d'assomption que Husserl développe tout particulièrement dans les dix premières pages d'Objets intentionnels (p. 150-160).

Afin d'entrer directement dans le vif du problème, considérons la thèse de Bolzano, la thèse $(\mathrm{A})$, comme une thèse non problématique. Pour résoudre le soi-disant paradoxe entre (A) et (B), il est plus simple - et c'est l'option choisie par Husserl - de travailler à une interprétation de (B) afin que (A) et (B) puissent figurer dans le cadre de la même théorie. La nécessité d'un concept qui contribuerait à l'élucidation de (B) est évoqué par Husserl de la manière suivante:

S'il est possible de parler dans tous les cas de l'objet de la représentation, alors il doit tout d'abord être possible d'identifier ou de différencier ces objets intentionnels (IG, p. 151).

On ne retrouve pas ici de contre-argument contre la thèse (B), mais plutôt une première mise en garde sur l'emploi de l'adjectif «intentionnel » appliqué aux objets de représentation. Si (B) doit être considérée comme vraie, alors il doit être possible d'identifier l'objet des représentations R1 et R2 comme étant le même objet, et ce, aussi bien pour des paires de représentations comme [le chien préféré de Bismarck] et [Tyras] que pour des paires de représentations comme [le chien préféré de Charlie Brown] et [Snoopy] ${ }^{13}$. Corrélativement, il doit être également possible de différencier les objets de $\mathrm{R} 1$ et R2 aussi bien pour des paires de représentation comme [Dante] et [Virgile] que pour des paires de représentations comme [le centaure Nessos] et [le centaure Chiron]. C'est là un point décisif de l'argumentation de Husserl dans Objets intentionnels, qui poursuit le double objectif de fournir une interprétation de (B) qui puisse en faire une thèse vraie sans toutefois contredire la thèse (A). Pour y arriver, on doit faire abstraction de l'existence ou de la non-existence de l'objet, ce qui signifie, dans le cas des paires de représentation dont nous venons de discuter, que l'identité des objets représentés doit être posée sous hypothèse:

Si l'on se demande maintenant ce que cela veut dire, dans le sens ici en question, que deux représentations - abstraction faite de l'existence ou de la nonexistence - représentent le même objet, on voit tout de suite qu'il ne peut s'agir d'identité que sous une hypothèse (IG, p. 151).

13. Ici et dans ce qui suit, j'emploie les crochets carrés ([, ]) pour introduire les contenus objectifs d'actes mentaux (aussi bien de jugements que de représentations). Les contenus subjectifs des actes mentaux seront introduits par des crochets pointus $(<,>)$. 
Plus tôt dans le texte, Husserl critiquait la distinction de Twardowski entre objet intentionnel et objet «vrai» ou réel ${ }^{14}$. Lorsqu'on parle d'objets intentionnels sans l'ajout modifiant "simple» (bloß intentionaler Gegenstand), alors on vise par là tous les objets, parmi lesquels on retrouve également les "vrais objets». Or, selon la citation précédente, on vise les objets dans la mesure où on fait abstraction de la question de leur existence. Cette "suspension du jugement » relativement à la question existentielle est ce qui permet de constater l'identité des objets des représentations [Snoopy] et [le chien préféré de Charlie Brown].

Avant d'aller plus loin, une remarque terminologique est nécessaire. J'emploie l'expression "suspension de jugement » afin de désigner une attitude psychologique dans laquelle, eu égard à certaines représentations, je mets entre parenthèses la question de leur objectualité. Lorsque je suspends mon jugement eu égard à l'objectualité des représentations [Snoopy] et [Le chien préféré de Charlie Brown], je ne statue pas sur le fait qu'il y a quelque chose de tel que Snoopy ou Charlie Brown. Snoopy et Charlie Brown sont alors pour moi de simples objets intentionnels (bloß intentionale Gegenstände). La suspension du jugement relativement aux représentations $\mathrm{R} 1$, $\mathrm{R} 2, \ldots, \mathrm{R} n$ est donc une suspension jugement par rapport à l'objectualité de $\mathrm{R} 1, \mathrm{R} 2, \ldots, \mathrm{R} n$.

Comment doit-on comprendre l'objet intentionnel au sens d'un objet ex hypothesi ? J'emploie ici l'expression "ex hypothesi» en rapport avec un objet ou un discours et ne veux par là rien dire d'autre qu'il s'agit là d'un objet tel que le suppose l'hypothèse (ou encore sous la portée de l'hypothèse). Lorsque je parle de discours ex hypothesi, je vise par là un ou des énoncés qui sont vrais ou faux sous la portée de l'hypothèse. Ainsi, un "objet ex hypothesi» est ce dont l'existence ou la non-existence est jugée sous assomption.

Afin de clarifier l'idée de l'hypothèse et de préciser ses fonctions, Husserl introduit un argument concernant l'aspect linguistique des hypothèses. Sans que cela ne soulève grande excitation, on dit souvent que Zeus est le plus grand des dieux olympiens. C'est là un énoncé d'identité tout à fait sensé qui, du reste, non seulement fait sens mais est également vrai, comme le confirmeront très certainement tous les connaisseurs de la mythologie de la Grèce ancienne. Cet énoncé est vrai, nous dit Husserl, mais il demeure incomplet tel qu'il est formulé. Dans mon énoncé «Zeus est le plus grand des dieux olympiens ", une partie de mon acte mental est passée sous silence. Pour communiquer la totalité de mon acte mental, je devrais dire: «Selon la mythologie de la Grèce ancienne, Zeus est le plus grand des dieux olympiens. » Ce jugement est vrai, car on juge par là ex hypothesi de l'existence des objets, l'hypothèse étant communiquée par l'ajout de l'antécédent «selon

14. Plus précisément, la distinction entre objets existant «vraiment» et objets existant «intentionnellement». Cf. Twardowski (1894), p. 21sq. 
la mythologie de la Grèce ancienne, ...». Ainsi, l'énoncé «Zeus est le plus grand des dieux olympiens» n'est qu'un pseudo-énoncé d'identité - un véritable énoncé d'identité devant inclure l'existence de ce qui est identifié. Il s'agit ici d'une identité mise entre parenthèses (dabingestellt). Car au sens absolu, l'énoncé d'identité est faux, mais comme il doit être lu avec l'ajout "selon la mythologie de la Grèce ancienne, ... ", la fonction naturelle de cet énoncé se situe dans le caractère impropre dans lequel il est généralement compris (IG, p. 151-152).

Comment communique-t-on une assomption husserlienne? Husserl ne donne pas de réponse univoque dans $I G$, mais on peut proposer la réponse suivante: les assomptions husserliennes sont communiquées par des phrases conditionnelles incomplètes (ou ouvertes) ${ }^{15}$. Stricto sensu, dans les exemples utilisés dans $I G$, l'assomption n'est toutefois pas communiquée par une conditionnelle ouverte. Pour la communiquer correctement, il faudrait dire: "Si la mythologie de la Grèce ancienne était vraie, alors ce serait le cas que...» Dans le cas qui nous intéresse ici, la conditionnelle est donc la suivante: "Si la mythologie de la Grèce ancienne était vraie, alors ce serait le cas que Zeus est le plus grand de tous les dieux olympiens ${ }^{16}$.»

Cette conception des assomptions mise de l'avant par Husserl soulève certaines questions, et notamment celle-ci: dans «Zeus est le plus grand des dieux olympiens ", on peut peut-être recourir à une certaine intuition générale de nos interlocuteurs pour le discours fictionnel afin de souligner la différence entre le discours propre et le discours ex hypothesi. Mais il y a naturellement des cas dans lesquels cette différence n'est pas remarquable du premier coup. Pensons notamment à des romans qui emploient des personnages réels dans des histoires fictives, par exemple Der Untergeher de Thomas Bernhard, où Glenn Gould, pendant les classes de maître d'Horowitz à Salzburg, aurait déraciné un hêtre de ses mains nues pour ensuite le scier en grosses bûches. Des lecteurs ayant différents degrés de connaissance à propos de la scène et des personnages décrits par Bernhard émettront nécessairement des assomptions différentes sur les énoncés du passage: certains lecteurs mettront l'ensemble de la réalité décrite dans le roman sous assomption, alors que d'autres ne mettront sous assomption qu'une partie du récit. En fait, la pratique de l'assomption par le lecteur dans les récits de fiction ou même dans la mythologie est à peu près aussi arbitraire que celle de l'auteur et, dans un cas comme dans l'autre, cette pratique ne suit généralement aucune règle déterminée d'avance ${ }^{17}$.

15. Que les assomptions soient communiquées par des énoncés conditionnels ouverts et non par des énoncés conditionnels complets, c'est ce que confirment certaines remarques de Husserl: les énoncés d'assomption sont "des assomptions à compléter " ( $z$ u ergänzende Assumption [en]) $(I G, 151)$ et elles ont des apodoses (Nachsätze) $(I G, 155)$.

16. Cf. IG, p. 151.

17. Il est d'ailleurs bien connu que les mythes de la Grèce ancienne prennent des formes différentes et souvent contradictoires selon les chronistes de l'époque qui rapportent ces mythes. 
Nous avons vu que Husserl conçoit l'assomption, et la suspension du jugement qui lui est corrélative, comme un moyen d'assurer la vérité d'énoncés d'identité dans des contextes où ces énoncés portent sur des objets qui n'existent pas. Dans la deuxième partie du texte $(I G$, p. 155 sq.) Husserl veut maintenant examiner de plus près la suspension du jugement qu'on opère lorsqu'on affirme par exemple que [le chien préféré de Charlie Brown] et [Snoopy] représentent le même objet. Husserl veut rendre clair le fait que la suspension du jugement est une méthode dont l'utilité scientifique est démontrée, et que ce n'est pas simplement une façon pratique d'empêcher des discussions à propos d'actions ou de personnages fictifs dans la littérature que de tomber dans des discussions sur le statut ontologique de telles actions ou figures. Il part de la thèse suivante:

Les lois formelles qui régissent la pensée sous assomption [sont] exactement les mêmes que celles qui sont valides pour une [pensée] soi-disant libre, c'està-dire une pensée restreinte par aucune condition ( $I G$, p. 155).

Cette thèse n'est pas en soi très problématique: je peux déduire de «Snoopy est le chien préféré de Charlie Brown » «il y a exactement un chien qui est le chien préféré de Charlie Brown ", de la même façon que je peux déduire de «Tyras est le chien préféré de Bismarck» «il y a exactement un chien qui est le chien préféré de Bismarck». Les énoncés qui sont vrais ou faux ex hypothesi sont régis par les mêmes lois logiques que les énoncés qui n'appartiennent pas au discours ex hypothesi, à la différence que le jugement que je communique par «il y a exactement un chien qui est le chien préféré de Charlie Brown» est un jugement émis sous assomption.

Une autre caractéristique de l'assomption husserlienne en 1894 est qu'elle ne doit pas nécessairement être émise consciemment. Je peux effectuer mentalement des prédications sur Snoopy et Charlie Brown, puis, à un certain point, je peux ne plus faire attention à la mise sous assomption de ces prédications. L'assomption n'a pas à être effectivement pensée afin que je me rapporte à Snoopy et Charlie Brown sous assomption. Il suffit que j'aie la disposition à juger sous assomption que Snoopy est le chien préféré de Charlie Brown pour que mon jugement d'identité soit considéré comme vrai ${ }^{18}$.

D'autre part, les récits de fiction dont la construction suit des règles précises sans jamais en déroger sont plutôt l'exception et semblent appartenir à un courant de la littérature bien postérieur à Husserl. Certains romans de Beckett, Le Dépeupleur par exemple, pourraient répondre à ces règles strictes.

18. L'assomption «en reste souvent à une excitation dispositionnelle et donne aux jugements conditionnés un caractère subjectif psychique par lequel ceux-ci nous apparaissent comme tels" $I G, 160$. Dans $I G$, le concept de disposition psychologique prend une place importante: les dispositions sont considérées comme des "possibilités réelles inconscientes" (unbewusste reale Möglichkeiten) (IG, p. 145); au moyen des dispositions, nous pouvons après-coup restreindre le cours de nos pensées par une assomption $(I G, \mathrm{p} .55)$. Cf. également $I G$, p. 156, 158, 160, 162, 165. Husserl défend une conception similaire des dispositions psychologiques dans $L U \mathrm{II} / 1$, p. 454. 
Par exemple, disons que je juge ex hypothesi que

(J1) Nessos est celui qui a enlevé Déjanire

et, partant de ce jugement, je juge ensuite

(J2) Déjanire est l'épouse d'Hercule;

(J3) Nessos est celui qui a enlevé l'épouse d'Hercule;

(J4) Hercule est le fils de Jupiter et d'Alcmène;

(J5) Hercule a vaincu l'Hydre;

Etc.

Dans cette chaîne de jugements, il se peut qu'à un certain moment je ne sois plus conscient de juger ex hypothesi, alors que, pourtant, tous les jugements sont formulés ex hypothesi. Husserl pense que les assomptions sont à la base de nos pensées sans fixation expresse, mais de manière évidente («ohne ausdrückliche Fixierung, jedoch selbstverständlich») (IG, p. 155). Ainsi, même lorsque je ne juge pas consciemment sous assomption que Hercule a vaincu l'Hydre, j'ai la disposition de restreindre mon jugement par l'assomption. Cette disposition suffit à elle seule à décrire la situation psychologique du sujet dans le discours ex hypothesi. On peut donc résumer ainsi: je ne suspends mon jugement par rapport à l'objectualité d'une représentation que de manière consciente. Mais que je ne le suspende pas ne signifie pas que je juge hors assomption et que je pose l'existence de ce qui est jugé. Il suffit que j’aie la disposition à le faire.

Cette réponse ne fait toutefois que déplacer le problème du côté de la nature des dispositions psychologiques, et, sur ce chapitre, Husserl ne s'aventure guère dans Objets intentionnels à élaborer pour elles des conditions d'individuation, alors qu'elles jouent pourtant un rôle clé dans sa conception des assomptions. En partant d'une de ses remarques sur les états dispositionnels d'un chercheur jugeant sous assomption, on peut toutefois donner une approximation de ce que sont probablement pour lui les dispositions en affirmant qu'elles forment le cadre à l'intérieur duquel le chercheur émet ses jugements. Qu'il soit ou non effectivement conscient des limites du cadre pour chaque jugement qu'il émet n'est pas nécessaire, il doit simplement l'avoir été au départ dans la formulation des axiomes:

La règle incassable - qui vit dans le chercheur bien instruit comme un complexe de dispositions effectives - de ne rien juger qui ne découle purement des axiomes, commande la validité des élaborations et détermine pratiquement les limites de l'espace logique (IG, p. 156).

Or, comme nous l'avons déjà mentionné, selon Husserl il en va en fiction tout comme en mathématique et en algèbre quant à l'utilisation de l'assomption. La pertinence interne d'un système mathématique n'est pas 
affectée par le fait qu'un chercheur déduit erronément de la définition posée sous assomption l'existence inconditionnelle des entités:

Ces impropriétés de la pensée, le fait que nous émettions d'innombrables jugements que, "au fond", nous ne visons pas vraiment - qu'il y ait un juger intérimaire comme il y a un représenter intérimaire et que l'un soit inséparable de l'autre, c'est là un des points centraux de l' «économie de la pensée ». La science comme réalisation spirituelle humaine ne peut être comprise sans elles (IG, p. 156).

Partant de l'exemple de la géométrie, Husserl revient sur le discours ex hypothesi: il est erroné de parler de l'existence du triangle de manière inconditionnelle. Non seulement parce que les triangles n' «existent» pas comme les animaux de compagnie ou les bicyclettes, mais particulièrement parce que "l'existence» des triangles en géométrie n'est comprise que comme une existence sous assomption. En fait, pense Husserl, toutes les propositions de la géométrie sont sous une assomption générale de la forme "nous posons une multiplicité que nous appelons espace, dont nous appelons les éléments des points qui ont les qualités $\mathrm{x}, \mathrm{y}, \mathrm{z},[\ldots]^{19}{ }^{»}$. Et sous cette assomption, on dira que:

Un carré rouge n'est pas une existence géométrique, un carré rouge et noir sur toute sa surface n'est pas une non-existence géométrique. Il s'agit plutôt d'existence ou de non-existence conditionnée de manière purement déductive, une conséquence logique nécessaire, sous l'hypothèse des fondements, là où il ne s'agit pas de la teneur existentielle expressément assumée de ceux-ci (IG, p. 159).

Ainsi, l'existence ou la non-existence (l'objectualité et l'inobjectualité) des formations géométriques (et des représentations de ces formations) est toujours conditionnée par l'hypothèse du fondement selon lequel de telles formations "existent" (sous l'hypothèse que leurs représentations soient objectuelles). Des énoncés sur l'objectualité des représentations en géométrie sont donc toujours formulés sous la condition d'une assomption. Le discours sous assomption s'applique donc aussi bien à la géométrie qu'au discours sur les fictions. Mais comment doit-on comprendre ce parallèle entre l'assomption dans le contexte géométrique et l'assomption dans le contexte fictionnel ? Est-ce que j'émets des jugements sur Jupiter de la même manière que j'émets des jugements sur des triangles isocèles? La position de Husserl dans le texte de 1894 est de répondre par l'affirmative. Or, on s'en aperçoit facilement, cette position aplanit une différence importante entre les objets fictifs et les objets abstraits : selon ce que dit Husserl, il devrait être possible de déterminer complètement les propriétés d'Emma Bovary de la même manière qu'il est possible de déterminer complètement les propriétés d'un triangle isocèle. Mais pourtant, le roman de Flaubert laisse au hasard

19. «Gesetzt, dass es einen Raum, eine Mannigfaltigkeit der so und so bestimmten (in den Grundlagen exakt definierten) Art gibt» (IG, p. 159). 
une quantité de détails sur Emma Bovary: son groupe sanguin et la taille de ses chaussures, par exemple. Alors que, nécessairement, les axiomes de la géométrie ne laissent rien au hasard quant aux objets qui sont déterminés par eux, est-ce à dire que, selon Husserl, les personnages de fiction seraient nécessairement indéterminés - et qu'ainsi Emma Bovary porterait des chaussures de longueur indéterminée et que ses caractéristiques sanguines ne répondraient à aucun groupe sanguin? Même si c'est là une conception des objets fictifs entérinée par plusieurs phénoménologues, elle n'est pas sans poser problème dans le contexte de la théorie de l'assomption de Husserl ${ }^{20}$.

\section{Quelques problèmes avec la solution de $\mathbf{1 8 9 4}$}

Comme le suggère Rollinger dans sa contribution au présent volume, si on peut dire en un certain sens que c'est à Husserl, et non à Meinong, que revient le mérite d'avoir découvert le domaine des assomptions dans l'école de Brentano, et ce, même quelques années déjà avant Meinong, il ne faut pas oublier que Meinong est le premier dans l'école de Brentano à s'être aventuré dans la sphère suppositionnelle, un geste que Brentano ne cessera d'ailleurs de réprouver ${ }^{21}$. Et, bien qu'elle s'engage ainsi sur un terrain que l'orthodoxie brentanienne considère déjà avec hostilité, il n'en demeure pas moins que la stratégie assomptive développée par Husserl en 1894 n'est pas encore tout à fait émancipée de la conception brentanienne du jugement, et ce, même si elle emprunte de manière efficace certains outils de la logique de Bolzano. Dans cette section, j’aimerais me pencher sur quelques difficultés relatives à la conception de l'assomption de 1894, notamment quant à cette position équivoque que prend Husserl entre Bolzano et Brentano, mais également sur deux points internes à la conception de Husserl, à savoir sa compréhension des énoncés conditionnels dans la communication des assomptions et le parallèle qu'il propose entre l'assomption en géométrie et celle opérée sur le discours fictionnel.

\subsection{Quel type de conditionnelle?}

Un premier problème dans la présentation que fait Husserl de l'assomption dans Objets intentionnels consiste en ceci que l'emploi des énoncés conditionnels comme exemples d'assomptions ne semble pas s'orienter sur la thèse qu'il prétend défendre. Dans le texte de 1894, ce sont des énoncés conditionnels contrefactuels qui sont employés pour formuler les assomptions. Or ces énoncés sont construits selon une forme particulière:

(Cond.1) Si $S$ était vrai, alors ce serait le cas que $P$

20. Cf. notamment Ingarden (1960), p. 120sq. et 261sq., Sartre (1940), p. 167sq. ou Meinong (1915), p. 168sq.

21. Et ce, dès 1889 (Brentano, 1889, p. 84) en réaction à la thèse des évidences suppositionnelles dans Meinong (1886). Cette critique se poursuit jusqu’à la fin de sa vie, notamment dans un manuscrit de 1916 contre la théorie de l'assomption de Meinong (Gegen die Annahme als besondere Grundklasse psychischer Beziehungen). 
où on présuppose que $S$ n'est pas vrai et que non- $P$, comme par exemple dans le cas suivant: si les Canadiens de Montréal avaient gagné la Coupe Stanley en 2009, alors ils n'auraient pas congédié leur entraîneur. Mais estce là vraiment ce que Husserl a en tête ? Car il pourrait bien avoir en tête un autre type de conditionnelle, une conditionnelle réelle:

(Cond. 2) Si $S$ est vrai, alors $P$

Dans le cas de (Cond. 2), on ne présuppose pas que $S$ n'est pas vrai (on ne présuppose pas que non-P), mais on ne présuppose pas non plus que $S$ est vrai (et donc que $P$ est vrai). Si on caractérise vraiment l'assomption comme la suspension du jugement, alors il semble, contre Husserl, que (Cond. 2) soit une meilleure description de l'assomption que (Cond. 1).

Gardons aussi à l'esprit que les conditions de vérité d'une conditionnelle contrefactuelle se laissent établir à l'aide d'une sémantique des mondes possibles. Or, si on veut appliquer une telle sémantique pour l'analyse des énoncés de Husserl, alors on ne quantifie plus sur le monde actuel, mais sur un monde possible, $M$, par exemple le monde de la mythologie de la Grèce ancienne ou celui dans lequel les Canadiens de Montréal ont gagné le tournoi 2009 de la Coupe Stanley. Dans ce contexte, la question se pose alors de savoir si la sémantique des mondes possibles est ce qui caractérise le mieux la stratégie assomptive développée dans Objets intentionnels, bien qu'en partant de ce qui est affirmé dans le texte, une interprétation du discours ex hypothesi en termes de quantification sur des mondes possibles apparaisse peu plausible. Cette impression est d'ailleurs renforcée par une remarque prima facie décisive de Husserl:

Ainsi, nous ne voulons autoriser le discours confus sur différents domaines d'existence, universes of discourse, sur différents "mondes » qui disposent différemment de l'existence et de la non-existence d'un même objet. Le "monde " du mythe, le monde de la poésie, le monde de la géométrie, le monde réel, ce ne sont pas des «mondes" du même droit. Il n'y a qu'une vérité et un monde, mais une multitude de représentations, de convictions mythologiques ou religieuses, d'hypothèses, de fictions [...] (IG, p. 159).

Même s'il me semble plus naturel de l'interpréter contre la lecture contrefactuelle, je ne pense pas que la citation précédente suffise en ellemême à exclure cette lecture: Husserl dit ici refuser de parler de "mondes du même droit» lorsqu'il est question du monde de la poésie et du monde réel. Or les mondes possibles ne sont justement pas des «mondes du même droit» que le monde actuel ${ }^{22}$. Pour neutraliser définitivement l'interprétation contrefactuelle, il faut donc trouver un appui supplémentaire.

22. En fait, dans des textes plus tardifs, Husserl se montre plutôt sympathique à l'interprétation contrefactuelle dans le cas des centaures et des ficta en général. $C f$. par exemple Hua XXIII, 515sq. et Hua XXXVI, p. 183sq. sur les mondes possibles des objets temporels effectifs (möglichen Welten tatsächlicher Zeitgegenstände). 
On trouve cet appui dans la conception husserlienne de l'assomption elle-même, selon laquelle le concept d'assomption découlerait de celui de position en mathématiques. On peut déjà voir que, dans la position d'axiomes en mathématiques, on ne part pas du présupposé que les entités dont il est question dans les axiomes n'existent pas, ou plutôt: on ne sait pas d'emblée qu'ils n'existent pas. L'exemple suivant devrait suffire pour s'en convaincre:

(J6) Il y a des carrés dont les côtés sont d'une longueur de $2 \mathrm{~cm}$.

est à comprendre en principe selon Husserl de la manière suivante:

(J7) Selon $P$, il y a des carrés dont les côtés sont d'une longueur de $2 \mathrm{~cm}$.

où $\mathrm{P}$ est une présupposition géométrique du type évoqué plus haut ${ }^{23}$. Or si on veut comprendre "selon $P$ » comme l'antécédent d'une conditionnelle contrefactuelle, on devrait alors comprendre (J8) de la manière suivante:

(J8) Si P était vrai, alors il y aurait des carrés dont les côtés ont une longueur de $2 \mathrm{~cm}$.

Mais de toute évidence (J8) n'est pas ce que les géomètres veulent dire en employant des axiomes, et les hellénistes ne veulent pas dire quelque chose d'analogue à (J8) lorsqu'ils affirment que Zeus est le plus grand des dieux olympiens. Et ce que Husserl veut dire en parlant d'assomption est bien plus proche d'une conditionnelle réelle que d'une conditionnelle contrefactuelle. Cette tendance se confirme dans les leçons de logique de 1896:

Lorsque le géomètre veut dire qu'une proposition ${ }^{24}$ générale subsisterait si l'existence des figures concernées était assurée, alors il dit, comme nous le disons dans la vie de tous les jours, "tous les $S$ seraient $P$ ", c'est-à-dire "supposant qu'il y en a [des S], alors il n'y en a aucun parmi eux qui ne serait pas $P »($ Hua M1, p. 184).

\subsection{Priorité du jugement sur la représentation}

La stratégie assomptive déployée par Husserl dans Objets intentionnels nous donne de bonnes raisons de croire qu'en 1894 sa position face à la théorie du jugement de Brentano n'est pas encore définitive. La terminologie employée par Husserl à certains endroits du texte donne quelques indices à cet effet: lorsque je juge «A est $\mathrm{B}$ », c'est l'existence de A qui est reconnue;

23. IG, p. 159: «Nous posons une multiplicité que nous appelons espace, dont nous appelons les éléments des points qui ont les qualités $\mathrm{x}, \mathrm{y}, \mathrm{z},[\ldots]$. »

24. Ici, ou bien Husserl veut dire un "état de choses» au lieu d'une "proposition ", ou bien le verbe «subsister»(bestehen) doit être remplacé par "valoir» (gelten) ou «être vrai» (wäre wahr). 
les objets sont «représentés ou jugés comme existants ${ }^{25}(I G$, p. 146); les actes s'exercent sur les objets «sur le mode de la représentation, de la reconnaissance ou du rejet» (IG, p. 146); l'acte "représente, reconnaît ou rejette son objet » (IG, p. 147); les jugements sur «l'existence ou la non-existence des objets " sont mis sous hypothèse $(I G$, p. 155). Mais l'indice le plus net est à trouver dans la nature de la stratégie elle-même: la mise sous assomption proposée par Husserl présuppose que les jugements sont essentiellement des beliefs en l'existence du représenté26. L'acte assomptif s'exerce à même un acte de jugement qua belief en l'existence du représenté, et cet acte de jugement est plus fondamental que l'assomption qui est exercée sur lui. Rappelons-nous le problème de départ duquel l'assomption est la solution: en partant de la théorie du jugement de Brentano, Twardowski affirmait que ma représentation <carré rond> devait avoir un objet, sans quoi je ne peux émettre le jugement négatif correct <il n'y a pas de carrés ronds $>$. Puisque, autrement dit, lorsque je porte le jugement <les carrés ronds n'existent pas>, je nie l'existence des carrés ronds, il y a quelque chose dont je nie l'existence. Dans un jugement, ce sont les objets eux-mêmes sur lesquels nous jugeons. Le jugement étant fondamentalement soit reconnaissance, soit rejet, alors c'est toujours l'existence de quelque chose qui est reconnue ou niée dans un jugement ${ }^{27}$. La théorie de l'assomption de Husserl nous évite certes cette conséquence fâcheuse de l'idée de Twardowski, mais elle ne remet pas en cause le fait que l'objectualité d'une représentation dépend du jugement que nous portons sur son objet: Husserl et Twardowski partent tous deux de la thèse centrale selon laquelle tous les jugements sont des convictions, des croyances en l'existence, et en tirent des conséquences différentes: pour Twardowski, parce que tous les jugements sont des convictions, toutes les représentations doivent avoir des objets, donc il y a quelque chose de tel que Zeus ou un carré rond; pour Husserl, parce que tous les jugements sont des convictions, on doit trouver un moyen d'assurer que deux convictions également valables mais contradictoires, comme $<$ Zeus est le plus grand de tous les dieux olympiens $>$ et $<$ Zeus n'existe pas $>$ puissent coexister. La solution consiste alors à introduire une distinction des deux jugements quant à leur mode assomptif. Le premier est un jugement conditionné (bedingtes Urteil), le second un jugement inconditionné (unbedingtes Urteil). Autrement dit, dans le texte de 1894 , la solution offerte par Husserl au problème des représentations sans objet n'est qu'une solution indirecte, qui s'attaque d'abord au problème de la validité de jugements portant sur des entités fictives ou

25. "Que nous nous représentions simplement Berlin ou que nous la jugions existante: c'est dans tout les cas Berlin elle-même " $(\mathrm{O} b$ wir Berlin blo $\beta$ vorstellen oder es als existierend beurteilen: es ist jedenfalls Berlin selbst) $(I G, 146)$.

26. Notons qu'on ne retrouve pas encore en 1894 de distinction explicite entre ce qui est affirmé par le jugement et la croyance ou conviction en l'existence du représenté, comme on la retrouvera thématisée dans les Recherches logiques, par exemple dans $L U$ II/1, p. 4 et ailleurs.

27. Cf. Twardowski (1894), p. 24. 
contradictoires. Non seulement le problème est formulé dans un cadre brentanien, mais sa solution ne sort pas non plus de ce cadre.

\subsection{La dette envers Bolzano: représentations et propositions}

Naturellement, cela n'empêche pas Husserl d'être bolzanien sur bien des plans dans ce texte: sur la question de la teneur objective des représentations et de la priorité systématique des représentations objectives sur les actes de représentation, sur le fait que les représentations objectives sont le contenu (la signification) des représentations subjectives (IG, p. 162), sur le rejet de la distinction entre objet réel et objet intentionnel au profit d'une distinction quant à la fonction logique des représentations, et sur le fait que la relation objectuelle d'une représentation relève de la teneur idéale, et non psychologique, de celle-ci (IG, p. 147). Ces positions sont parfaitement étrangères à celles de Brentano et de ses autres étudiants à cette époque ${ }^{28}$. Et ce n'est aucunement spéculer que d'affirmer que Husserl soutient, en 1894, la thèse que la représentation objective [carré rond] est une représentation sans objet, autrement dit un concept dont l'extension ne contient aucun individu. Mais on ne doit pas se tromper: cette position est un des deux termes du problème des représentations sans objet, ce n'en est aucunement la solution. Et en fait, la dette de Husserl envers Bolzano dans ce texte de 1894 est plus limitée qu'on peut être porté à le croire à première vue. $\mathrm{Si}$, quant aux représentations, Husserl suit Bolzano en attribuant aux représentations objectives une priorité systématique sur les représentations subjectives, cette allégeance ne s'étend toutefois pas au domaine propositionnel. Les assomptions sont des actes mentaux qui s'exercent sur d'autres actes mentaux, et non pas sur des propositions, et ces assomptions n'ont pas, prima facie, de pendant objectif comme les représentations subjectives ont leur pendant dans les représentations objectives. Si plusieurs passages du texte de 1894 nous permettent de croire que Husserl distingue au moins terminologiquement entre une proposition au sens de Bolzano et un jugement, aucun de ceux-ci ne nous permet de statuer précisément ce qu'il entend par proposition ${ }^{29}$. Et il semble qu'en 1894 Husserl comprenait par là quelque chose d'assez semblable à la conception qu'il avait des propositions dans sa discussion de la logique de Schröder, et qu'il n'avait pas encore assimilé complètement le concept bolzanien de proposition ${ }^{30}$. La

28. Évidemment, Twardowski (op. cit., p. 17) voit bien l'importance de la représentation objective de Bolzano, mais il ne parvient à aucun moment dans cet ouvrage à l'émanciper de l'acte de représentation. Elle demeure ainsi un contenu psychique.

29. En fait, on sait que Husserl, même pendant la rédaction des Prolégomènes, n'était pas parfaitement au clair sur la distinction entre jugement, proposition et état de choses. $C f$. Fréchette (2009, 2009a, 2009b). Sur les rapports entre Bolzano et le jeune Husserl, cf. la contribution de Künne dans le présent numéro.

30. Il y a potentiellement une certaine parenté entre le concept de proposition défendu par Husserl à cette époque et celui de Herbart, $c f$. Herbart (1850), p. 94, bien qu'il manque à ce propos des preuves concrètes ( $c f$. Rang (1979), p. XXIV). 
preuve en est que l'explicitation du contenu des représentations qu'il propose (IG, p. 160sq.) et la théorie formelle des inférences qui est en jeu dans sa conception de l'assomption - théorie selon laquelle les formes d'inférence ne peuvent être rien d'autre que des déductions hypothétiques et que, conséquemment, ce qui semble être des jugements catégoriques est en fait des jugements hypothétiques - sont à interpréter dans le cadre des deux textes de Husserl sur la logique de Schröder de 1891, et aucunement en relation avec la théorie des inférences de Bolzano ${ }^{31}$ : autant dans Objets intentionnels que dans ses deux articles de 1891 sur la logique de Schröder, la préoccupation de Husserl est de montrer que les déductions partant d'axiomes ne sont pas des positions absolues d'existence (des jugements catégoriques), mais des jugements hypothétiques. Cette préoccupation est étrangère à la théorie de Bolzano qui définit une inférence à partir de la variabilité de certaines représentations en soi comprises dans les prémisses et la conclusion ${ }^{32}$. De toute évidence, la logique des variations de Bolzano n'est ni au programme des articles de 1891 ni dans celui de la théorie des assomptions de $1894^{33}$.

Examinons comment Husserl fait intervenir sa conception des inférences dans sa théorie de l'assomption. En effet, il considère qu'un jugement universel affirmatif comme

(J9) Les chevaux ont des sabots

31. Cf. le compte rendu de 1891 de la logique de Schröder par Husserl, tel que reproduit dans Hua XXII, p. 3sq. et ici particulièrement p. 35, ainsi que «Der Folgerungskalkül und die Inhaltslogik» de la même année, reproduit dans Hua XXII, p. 44sq.

32. Cf. WL II, 199: «[Il y a] dans les propositions [prémisses] A, B, C... [et conclusions] $\mathrm{M}, \mathrm{N}, \mathrm{O} \ldots$ certaines parties qui peuvent être considérées comme variables, avec pour conséquence que tout ensemble de représentation qui, à la place [des représentations] $\mathrm{i}, \mathrm{j}, \ldots$...rend vrai toutes les A, B, C... rend aussi vrai toutes les $\mathrm{M}, \mathrm{N}, \mathrm{O} \ldots$... ([es gibt] in den Sätzen A, B, C... M, $N$, O gewisse Theile [...], die als veränderlich betrachtet werden können, mit dem Erfolge, daß jeder Inbegriff von Vorstellung, der an der Stelle der $i, j, \ldots$ die sämmtlichen $A, B, C, \ldots$ wahr macht, auch die sämmtlichen $M, N, O$... wahr mache).

33. Sur l'opinion fort réservée de Husserl concernant la théorie des inférences de Bolzano, voir l'esquisse d'une préface aux Recherches logiques, telle que reproduite dans Hua $X X / 1$, particulièrement p. 310. Cf. également Beyer (1996), p. 37, pour une interprétation de ce passage. Afin de bien voir ce qui sépare la conception des inférences de Husserl de celle de Bolzano relativement au problème qui nous intéresse ici, prenons l'exemple suivant: pour pouvoir, de la proposition (1) exprimée par «il n'y a pas de polygones ronds ", déduire la proposition (2) qu'exprime «il n'y a pas de carrés ronds " sans s'engager à affirmer que (3) les carrés ronds sont une sorte de polygones ronds, Bolzano propose de faire varier la représentation [rond] dans (1) et (2), par exemple par la représentation [comprenant deux diagonales]. Si la paire de variantes objectuelles est vraie, comme c'est ici le cas, alors l'inférence de départ est valide. Husserl résout le problème en faisant l'assomption de polygones ronds, et donc (3) est vraie sous assomption pour Husserl, alors que (3) demeure fausse même si on peut déduire (2) de (1) pour Bolzano. Voir Hua XXII, p. 35 et Bolzano, WL I, p. 303sq. ainsi que Fréchette (2009a), p. 174sq. 
manifeste en fait une proposition de la forme [si quelque chose est un A, alors c'est un B], ou, pour la formuler complètement [si quelque chose est un cheval, alors cela a des sabots $]^{34}$. Cette conception de la proposition affirmative universelle comme ayant la forme d'une proposition hypothétique se marie remarquablement bien avec l'idée des dispositions psychologiques à l'assomption dans le cas des jugements: que mon jugement que tous les $\mathrm{A}$ sont $\mathrm{B}$ soit ou non porté sous assomption, et dans le second cas, que j'aie ou non la disposition à le porter sous assomption, «tous les $\mathrm{A}$ sont $\mathrm{B}$ » est de toute façon un jugement qui a la forme propositionnelle [si quelque chose est un A, alors c'est un B]. Autrement dit, dans Objets intentionnels, l'assomption et la disposition assomptive auraient un corrélat du côté de la forme de la proposition, qui est la proposition hypothétique au cœur de la théorie formelle des inférences de Husserl. Le rejet implicite de la théorie bolzanienne des inférences et la forme de la proposition préconisée par Husserl dans ce contexte suffisent à nous convaincre que ce corrélat n'est pas la proposition en soi bolzanienne ${ }^{35}$.

\subsection{Géométrie et fiction}

Une quatrième difficulté de la théorie de l'assomption de Husserl que nous avons déjà évoquée plus tôt dans le texte tient en ceci qu'elle compare de manière indifférenciée les assomptions sur les objets mythologiques avec les assomptions en géométrie. Il y a bien sûr une certaine analogie entre ces deux types d'assomption, mais, dans les deux cas, celles-ci sont employées à des fins fort différentes. En géométrie, les assomptions supportent des objectifs scientifiques, ce qui est rarement le cas des assomptions que nous portons relativement aux objets de discours fictionnels comme Zeus, Chiron ou Snoopy. Autrement dit, si on respecte le parallèle suggéré par Husserl de

34. Cf. IG, 154. C'est naturellement a fortiori le cas pour un jugement, comme le manifeste l'énoncé: "Les centaures ont des sabots."

35. Dans plusieurs passages d'Objets intentionnels, on peut s'apercevoir de la présence silencieuse de la logique de Schröder au sein des préoccupations de Husserl, notamment par le biais de certains exemples et expressions employés par Husserl dans ce texte. Qu'on pense à l'explicitation du contenu de représentation exprimé par "un lion» (ein Tier vom Katzengeschlecht, etc. Cf. IG, 160 et Schröder (1890, p. 231)) ou à l'idée de la décomposition du contenu d'une représentation en une chaîne de prédications (IG, 161: "wir ,lösen' die einheitliche Attribution in eine Kette von Prädikationen ,auf' ", ou encore à l'idée de Venn (1881, p. 128sq.) entérinée par Schröder (ibid., p. 243) des «universes of discourse» à laquelle Husserl dit s'opposer $(I G, 159)$. Notons au passage qu'à peu près au même moment où Husserl écrivait Objet intentionnels, il discutait avec Venn (Hua BW7, p. 265-268) précisément de sa recension de Schröder de 1891. Pour une évaluation récente des débats entre Schröder, Husserl et Frege, voir Kienzler (2007). Mentionnons également, bien qu'elles soient nettement plus marginales, les lectures de Kern (1888) et de Sigwart (1889) par Husserl qu'on retrouve indirectement dans Objets intentionnels, comme l'attestent les notes marginales reproduites dans Hua XXII, 457 sq. L'exemple "Zeus est le plus grand des dieux olympiens» est d'ailleurs repris de Kern (1888), p. 93sq. 
manière stricte, il devrait être possible en principe d'établir une science de la fiction qui, tout comme la géométrie pour les formations de son domaine, formulerait des propositions et des théorèmes exacts sur ce qui relève de son domaine. En suivant un parallèle exact entre l'assomption géométrique et l'assomption mythologique, on se doit de défendre la conception suivante: les objets du discours de la géométrie, tout comme les objets du discours fictionnel, sont entièrement déterminés par l'assomption. Cela revient à dire que lorsque nous jugeons sous assomption que Thésée a vaincu le Minotaure, la prémisse assomptive "selon la mythologie grecque...» contiendrait tout ce qu'il y a à savoir sur la victoire de Thésée sur le Minotaure, et ce, de la même façon que lorsque nous jugeons «les triangles sont des polygones de trois côtés", la prémisse assomptive "selon les axiomes de la géométrie..." contient tout ce qu'il y a à savoir sur les triangles. Dans un cas comme dans l'autre, il n'y aurait aucune marge de manœuvre, aucune interprétation possible, aucune indétermination. La question de savoir si Thésée fait partie des donneurs sanguins universels serait dans ce contexte une erreur de catégorie du même genre que la question de savoir quelle est la couleur de triangle la plus répandue. Dans les deux cas, il est impossible de répondre, car les prémisses assomptives ne présupposent rien sur ces questions $^{36}$.

Si cette interprétation rigide de l'assomption sur le discours fictionnel au sens de l'assomption sur le discours géométrique est formellement correcte et que, du reste, elle reproduit fidèlement les intentions de Husserl dans Objets intentionnels, elle va à l'encontre d'une intuition généralement partagée sur le discours fictionnel, à savoir qu'il y a là place à l'erreur, à l'inexactitude, à la contradiction, au contresens et à l'imprécision, bref à tout ce qui est par principe exclu dans la formulation des assomptions en géométrie. Cette divergence fondamentale entre la fiction et la géométrie s'étend aussi aux langages dans lesquels ils sont idéalement formulés: les axiomes et les théorèmes de la géométrie s'expriment idéalement dans un langage formel, alors que les assomptions et les propositions du discours fictionnel n'ont pas à répondre - et répondent rarement ! — à cette exigence.

36. Soulignons au passage la position de Husserl quant au fait de considérer les objets abstraits (comme les objets géométriques) comme étant posés sous assomption, et non obtenus par abstraction et généralisation à partir d'une représentation intuitive, comme le conçoivent Brentano et beaucoup de ses étudiants. Si on élargissait cette position aux objets idéaux de la logique (les représentations en soi et les propositions), on aurait une position selon laquelle les représentations en soi et les propositions ne sont pas des abstractions de représentations subjectives ou de jugements (la lecture brentanienne de Bolzano par Twardowski, par exemple, $c f$. Twardowski, op. cit. p. 17), sans toutefois appartenir à un domaine d'objets idéaux au sens des propositions en soi de Bolzano ou des Gedanken de Frege. Si, comme le suggère Rollinger (1999, p. 152), Husserl peut sembler proche de cette position dans Objets intentionnels, ce n'est pas celle qu'il adoptera dans les Recherches logiques. 
Afin de conserver ce qu'il y a d'éclairant dans le parallèle avancé par Husserl entre l'assomption sur le discours fictionnel et l'assomption en géométrie, je suggère de l'interpréter dans un sens plus souple. Une façon d'aborder ce parallèle est d'y voir une simple analogie, un tertium comparationis. En d'autres termes, on peut voir les assomptions de la géométrie et les assomptions dans le discours sur des objets fictifs comme deux types d'assomptions, qui appartiennent au genre général de l'assomption. Les assomptions du mathématicien dans la pensée scientifique ont ceci de commun avec mes assomptions sur Pégase que ce sont des assomptions, et rien de plus. La différence entre ces deux genres d'assomption serait simplement que l'assomption portée sur des jugements sur les objets et les personnages de la mythologie grecque, ou de la littérature en général, n'a pas à répondre aux mêmes exigences que les assomptions de la géométrie. Bien qu'il y ait au moins un endroit dans Objets intentionnels qui parle en faveur d'une telle interprétation, le concept d'assomption n'y est introduit nulle part comme tertium comparationis ${ }^{37}$. À la lumière de la précédente discussion, il est clair cependant que Husserl aurait gagné à favoriser une interprétation plus souple du parallèle.

\section{Remarques finales}

Nous avons vu dans ce qui précède que Husserl développe en 1894 un concept fort original d'assomption afin de proposer une solution au problème des représentations sans objet. Au sens strict - qui est le seul valable n'existent ni Zeus, ni Emma Bovary et Snoopy, ni les carrés et les triangles, point final. Mais le discours sur les objets géométriques, tout comme le discours fictionnel, est constitué de telle manière que l'existence de tels personnages et de telles formations géométriques est posée sous assomption. Husserl peut donc faire l'économie de ce qu'on pourrait appeler le jusqu'auboutisme brentanien de Twardowski, consistant à dire qu'il doit bien y avoir un objet dont nous nions l'existence lorsque nous nions l'existence de la montagne d'or, de Zeus ou des carrés ronds ${ }^{38}$.

Comme telle, cette solution n'est cependant pas idéale, comme je me suis efforcé de le montrer. Mais ce qui fait l'intérêt de la position de 1894, et plus spécifiquement du texte Objets intentionnels, c'est qu'il est en quelque sorte le laboratoire d'influences distinctes sur un problème central à la fois en logique et en psychologie descriptive: le problème des représentations sans objet. On retrouve dans ce texte les préoccupations de Husserl sur les inférences et sa discussion avec Schröder de 1891, on retrouve aussi une remise en question des thèses fondamentales brentanienne sur la classification des actes et sur les conséquences d'une conception essentiellement

37. Cf. IG, p. 160, où Husserl parle d'un côté d'une assomption fictive, et de l'autre d'une assomption scientifique.

38. Cf. Twardowski, op. cit., p. 24. 
psychologique du contenu des représentations, et on retrouve également une première mise à profit des ressources de la logique de Bolzano, principalement autour des thèses qu'il y a des représentations sans objet et que les représentations subjectives ont une teneur idéale, ce que Husserl appelle déjà dans ce texte la Bedeutung, qui n'est rien d'autre que la représentation objective de Bolzano.

On doit noter également que le concept d'assomption de 1894, au moins tel qu'il est compris dans Objets intentionnels, ne trouve aucune application directe dans les Recherches logiques. Husserl ne discute pas la question de l'assomption de manière explicite dans les $R L$, mais il y introduit une distinction qu'on peut rattacher aux réflexions sur l'assomption de 1894. En effet, la distinction entre actes positionnels et actes non positionnels des $R L$ rappelle celle entre les jugements inconditionnés et les jugements sous assomption présentée dans $I G$ :

[I]ls [=les actes positionnels] sont [...] des visées d'existence; ils sont, soit des perceptions sensibles, soit des perceptions au sens plus large d'appréhensions présomptives d'existence en général, soit d'autres actes qui [...] le visent $[\ldots]$ comme existant.[...] Les autres actes [=non positionnels] laissent en suspens l'existence de leur objet; l'objet considéré objectivement peut exister, mais, en eux-mêmes, il n'est pas visé selon le mode existentiel [...] (LU II/1, p. 499; $R L$ II/2, p. 292).

Partant de cette brève description, on peut bien voir la différence entre les actes positionnels et les actes non positionnels, et dans quelle mesure on peut reconnaître une parenté entre les jugements sous assomption et les actes non positionnels. Dans l'assomption telle qu'elle est comprise en 1894, l'être de l'objet est également mis de côté; des jugements comme celui que manifeste "Zeus est le plus grand des dieux olympiens» sont habituellement déterminés par ce qui a été décrit ici comme la suspension du jugement. C'est pour cette raison que la thèse selon laquelle la distinction entre des jugements inconditionnés et les jugements ex hypothesi trouverait son pendant dans la distinction entre actes positionnels et actes non positionnels, telle que présentée dans les $R L$, est une thèse attrayante ${ }^{39}$. Mais il y a une différence importante entre les deux conceptions, et elle repose précisément sur le fait qu'en 1894 c'est en vertu d'assomptions, donc d'une classe d'actes autonome et différente à la fois des représentations et des jugements, que je me rapporte à un objet lorsque je me représente une montagne d'or ou Zeus. Dans la distinction des Recherches logiques, la distinction a lieu sur un plan beaucoup plus fondamental, à savoir les genres de qualité d'acte (positionnels/non positionnels), et sur ce plan, elle concerne tous les actes objectivants exactement de la même manière, donc les actes nominaux (les «représentations») et les actes propositionnels (les «jugements»). Du coup,

39. C'est la thèse que soutient notamment Pietersma, op. cit., p. 315. 
on ne fait pas intervenir, comme en 1894, un type ou une classe d'actes particuliers en vertu desquels les représentations sans objet sont des représentations qui se dirigent quand même sur des objets. Il y a donc une différence fondamentale entre l'assomption comme acte psychique, dont la nature est différente des représentations et des jugements, et le caractère non positionnel des actes: ce dernier, contrairement au premier, n'est tout simplement pas un acte $^{40}$.

Après les Recherches logiques, Husserl se penche à nouveau sur le problème de l'assomption, comme on peut le voir à partir des cours qu'il a professés à Göttingen jusque dans les années dix ${ }^{41}$. Brièvement, on doit noter qu'il ne semble plus entériner le parallèle strict entre l'assomption en géométrie et l'assomption pour le discours fictionnel ${ }^{42}$. Toutefois, ce qui est déterminant pour notre propos, c'est que l'assomption après les Recherches logiques demeure un phénomène distinct de ce qui rend certains actes non positionnels ${ }^{43}$. Autrement dit, malgré quelques points de détails problématiques dont nous avons discuté, il y a une certaine constance dans le traitement de l'assomption allant d'Objets intentionnels aux Recherches logiques, puis aux cours de Göttingen. Mais ce n'est qu'en 1894 que Husserl voit dans le phénomène de l'assomption une solution au problème des représentations sans objet. La solution qu'il proposera à ce problème dans les Recherches logiques s'émancipera des actes assomptifs. Toutefois, ce qu'on doit retenir, c'est qu'en 1894, Husserl développe les linéaments d'une théorie fort originale de l'assomption, et c'est avec raison qu'il peut écrire en 1902 à Meinong avoir déjà eu exactement les mêmes réflexions que lui à ce sujet plusieurs années auparavant. Il a également raison lorsqu'il dit qu'on ne trouve pas de phénoménologie du jugement hypothétique dans les Recherches logiques. Mis à part la difficulté du problème, une des raisons à cela est

40. Cette distinction entre acte positionnel et non positionnel est essentielle à la conception de l'intentionnalité défendue par Husserl dans les Recherches logiques. Pour un exposé de la conception de l'intentionnalité dans les $R L, c f$. Künne (1986). Je discute également de cette distinction dans Fréchette (2009a) et (2009b).

41. Cf. par exemple Hua XXX, p. 84 Hua XXIV, p. 47sq. et particulièrement Hua $X X V I$, p. $74 s q$.

42. Dans le cours de 1906 (Hua XXIV, p. 47sq.), Husserl distingue d'ailleurs très nettement la constitution d'hypothèses pour ce qui relève de l'empirie (le domaine de la psychologie), de la constitution d'hypothèses pour ce qui relève des objets idéaux (le domaine des mathématiques et de la logique).

43. Dans un cours de 1908-1909 (Hua M6, p. 195) Husserl suggère, visiblement en écho à On Denoting de Russell, qu'en réaction à l'énoncé «le présent empereur de France est aveugle » (exemple de Husserl), je peux émettre le jugement "cela n'est pas vrai, c'est faux " sous assomption, et dans ce jugement, je conteste la prédication comprise dans l'énoncé, et cette contestation est une position. La distinction entre assomption et caractère positionnel est également bien mise en évidence par Heinrich (1910), p. 90sq. qui se base presqu'essentiellement sur les cours de Husserl qu'il a suivis à Göttingen pendant cette période. Notons que Husserl a personnellement contribué à améliorer cette thèse qu'il a dirigée, $c f$. Schuhmann (1977), p. 127 et Hua $X X / 2$, p. 343sq. 
à trouver à mon avis dans le fait que cette théorie du jugement hypothétique n'est pas nécessaire à la structure des Recherches logiques, comme ne l'est pas la théorie de l'assomption pour le propos développé dans Objets intentionnels ${ }^{44}$.

\section{Bibliographie}

\section{Abréviations}

RL Husserl, Edmund, Recherches logiques, 3 tomes, trad. fr. par L. Kelkel, R. Schérer, H. Élie, Paris, PUF, 1957.

IG _ - «Ursprüngliche Druckfassung der Abhandlung "intentionale Gegenstände" von Husserl», in Karl Schuhmann, «Husserls Abhandlung "intentionale Gegenstände". Edition der ursprünglichen Druckfassung ", in Brentano Studien, III, 1990-1991, pp. 142-176.

LU -, Logische Untersuchungen, 2 tomes, Halle, Max Niemeyer, 19001901.

Hua M1 -, Logik. Vorlesung 1896, E. Schuhmann (dir.), Kluwer, Dordrecht, 2001.

Hua M6 -, Alte und neue Logik. Vorlesung 1908/09, E. Schuhmann (dir.), Kluwer, Dordrecht, 2003.

Hua BW1 -, Briefwechsel, Band 1: Die Brentanoschule, K. et E. Schuhmann (dir.), Kluwer, Dordrecht, 1994.

Hиа BW2 -, Briefwechsel, Band 2: Die Münchener Phänomenologen, K. et E. Schuhmann (dir.), Kluwer, Dordrecht, 1994.

Hua BW7 -, Briefwechsel, Band 7: Wissenschaftlerkorrespondenz, K. et E. Schuhmann (dir.), Kluwer, Dordrecht, 1994.

Hua XXII -, Aufsätze und Rezensionen (1890-1910), B. Rang (dir.), Den Haag, Martinus Nijhoff, 1979.

Hua XXIII —, Phantasie, Bildbewusstsein, Erinnerung. Zur Phänomenologie der anschaulichen Vergegenwärtigungen. Texte aus dem Nachlass (18981925), E. Marbach (dir.), Den Haag, Martinus Nijhoff, 1980.

Hua XXIV -, Einleitung in die Logik und Erkenntnistheorie (1906-1907), U. Melle (dir.), Den Haag, Martinus Nijhoff, 1984.

Hua XXVI —, Vorlesungen über Bedeutungslehre. Sommersemester 1908, U. Panzer (dir.), Den Haag, Martinus Nijhoff, 1987.

Hua XX/1 -, Logische Untersuchungen. Ergänzungsband. Erster Teil. Entwürfe zur Umarbeitung der VI. Untersuchung und zur Vorrede für die Neuauflage der Logischen Untersuchungen (Sommer 1913), U. Melle (dir.), Dordrecht, Kluwer, 2002.

Hua XX/2 -, Logische Untersuchungen. Ergänzungsband. Zweiter Teil. Texte für die Neufassung der VI. Untersuchung: Zur Phänomenologie des

44. Une première ébauche de ce texte a été présentée à Salzburg, en janvier 2008, dans le séminaire sur Husserl de Johannes Brandl, que je remercie d'ailleurs pour ses commentaires constructifs, tout comme Arek Chrudzimski et Robin Rollinger pour leurs riches interventions. Je remercie le FQRSC pour son soutien financier. 
Ausdrucks und der Erkenntnis (1893-1994; 1921), U. Melle (dir.), Dordrecht, Springer, 2005.

Hua XXX -, Logik und allgemeine Wissenschaftstheorie. Vorlesungen Wintersemester 1917-1918. Mit ergänzenden Texten aus der ersten Fassung von 1910-1911, U. Panzer (dir.), Dordrecht, Kluwer, 1996.

Hua XXXVI - , Tranzendentaler Idealismus. Texte aus dem Nachlass (1908-1921), R. Rollinger (dir.), Dordrecht, Kluwer, 2003.

Hua XL -, Untersuchungen zur Urteilstheorie. Texte aus dem Nachlass (18931918), R. Rollinger (dir.), Dordrecht, Springer, 2009.

WL Bolzano, Bernard, Wissenschaftslehre, volume I-IV, Sulzbach, Seidel'sche Buchhandlung, 1837 (réimpr. Aalen, Scientia, 1981).

Beyer, Christian. Von Bolzano zu Husserl. Eine Untersuchung über den Ursprung der phänomenologischen Bedeutungslehre, Dordrecht, Kluwer, 1996.

Brentano, Franz. Vom Ursprung sittlicher Erkenntnis, Leipzig, Duncker \& Humblot, 1889.

Cavallin, Jean. Content and Object. Husserl, Twardowski and Psychologism, Dordrecht, Kluwer, 1997 ( $1^{\text {re }}$ éd. Stockholm, 1990).

English, Jacques. "Présentation", in Edmund Husserl \& Kazimierz Twardowski, Husserl-Twardowski. Sur les objets intentionnels (1893-1901), Paris, Vrin, 1993, p. 9-84.

Fisette, Denis. "Représentations. Husserl critique de Twardowski », in D. Fisette et S. Lapointe (dir.), Aux origines de la phénoménologie: Husserl et le contexte des Recherches logiques, Paris/Québec, Vrin/Presses de l'Université Laval, 2003, p. 61-91.

Fréchette, Guillaume. «De la proposition à l'état de choses. Husserl, lecteur de Bolzano ", in Catégories logiques et catégories ontologiques, D. Seron, et al. (dir.), Genève, Droz, 2009 (à paraître).

—_. (2009a). Gegenstandslose Vorstellungen. Bolzano und seine Kritiker, Academia, Sankt-Augustin (sous presse).

—. (2009b). "L'intentionnalité et le caractère qualitatif des vécus. Husserl, Brentano et Lotze» (à paraître).

Heinrich, Erich. Untersuchungen zur Lehre vom Begriff, Göttingen, Kästner, 1910.

Herbart, Johann Friedrich. Sämtliche Werke (G. Hartenstein, dir.), $1^{\mathrm{er}}$ volume, Schriften zur Einleitung in die Philosophie, Leipzig, Leopold Voss, 1850.

Hickerson, Ryan. The History of Intentionality. Theories of Consciousness from Brentano to Husserl, Continuum, Londres, 2007.

Ingarden, Roman. Das literarische Kunstwerk, Tübingen, Niemeyer ( $2^{\mathrm{e}}$ édition augmentée), 1960.

Kern, Franz. Die deutsche Satzlehre. Eine Untersuchung ibrer Grundlagen, Berlin, Nicolaische Verlags-Buchhandlung, 1888.

Kienzler, Wolfgang. " "Man sollte mit Subjekt und Prädikat in der Logik aufräumen”. Die Schröder-Frege-Husserl-Kontroverse », in R. Bübner, G. Hindrichs (dir.), Von der Logik zur Sprache. Stuttgarter Hegel-Kongreß 2005, Stuttgart, Klett-Cotta, 2007, p. 256-271.

Künne, Wolfgang. "Edmund Husserl: Intentionalität», dans Speck, J. (dir.): Grundprobleme der großen Philosophen, Philosophie der Neuzeit IV, Göttingen, Vandenhoeck \& Ruprecht, 1986, p. 165-215. 
- «Fiktion ohne fiktive Gegenstände: Prolegomenon zu einer Fregeanischen Theorie der Fiktion ", in J. Brandl, A. Hieke, et P. Simons, (dir.): Metaphysik. neue Zugänge zu alten Fragen, Sankt Augustin, Academia, 1995, p. 141-161. Reproduit avec modifications dans M. Reicher (dir.), Fiktion, Wabrheit, Wirklichkeit. Philosophische Grundlagen der Literaturtheorie, Mentis, Paderborn, 2007, p. 54-72.

Meinong, Alexius. "Zur erkenntnistheoretischen Würdigung des Gedächtnisses », in Vierteljahrsschrift für wissenschaftliche Philosophie, vol. 10, 1886, p. 7-33.

—. Über Möglichkeit und Wahrscheinlichkeit. Beiträge zur Gegenstandstheorie und Erkenntnistheorie, Leipzig, Barth, 1915.

Münch, Dieter. Intention und Zeichen. Untersuchungen zu Franz Brentanos und Edmund Husserls Frühwerk, Frankfurt, Suhrkamp, 1993.

Naberhaus, Thane Martin. «Does Husserl Have an Argument against Representationalism?", in Grazer Philosophische Studien, vol. 73, 2006, p. 43-68.

Philipse, Herman. "The Concept of Intentionality. Husserl's Development from the Brentano Period to the Logical Investigations ", in Philosophy Research Archives, vol. 12, mars 1987, 1987, pp. 293-328.

Pietersma, Henry. "Husserl's Concept of Existence» in Synthese, vol. 66, 1986, p. 311-328.

Rang, Bernard. "Einleitung des Herausgebers », in Hua XXII, 1979, p. IX-LVI.

Reinach, Adolf. "Die obersten Regeln der Vernunftschlüsse bei Kant », in Kant Studien 16, 1911, p. 214-233.

Rollinger, Robin D. "Meinong and Husserl on Assumptions» in Axiomathes, vol. 1-2, 1996, p. 89-102.

- Husserl's Position in the School of Franz Brentano, Dordrecht, Kluwer, 1999.

Sartre, Jean-Paul. L'imaginaire. Psychologie phénoménologique de l'imagination, Paris, Gallimard, 1940.

Schröder, Ernst Vorlesungen über die Algebra der Logik (Exakte Logik), volume 1, Leipzig, Teubner, 1890.

Schuhmann, Karl. Husserl-Chronik. Denk- und Lebensweg Husserls, Den Haag, Martinus Nijhoff, 1977.

—. "Husserls Abhandlung “intentionale Gegenstände”. Edition der ursprünglichen Druckfassung », in Brentano Studien, III, 1990, pp. 137-176.

- «Intentionalität und intentionaler Gegenstand beim frühen Husserl» in C. Leijenhorst et P. Steenbakkers (dir.), Karl Schubmann. Selected Papers on Phenomenology, Dordrecht, Kluwer, 2004, p. 119-136 [publié originalement en 1991].

Schuhmann, Karl et Elisabeth Schuhmann. "Husserls Manuskripte zu seinem Doppelvortrag von 1901 », in Husserl Studies, vol. 17, 2001, p. 87-123.

Sepp, Hans-Reiner (dir.). Edmund Husserl und die phänomenologische Bewegung: Zeugnises in Text und Bild, Freiburg, Alber, 1988.

Sigwart, Christoph. Logik, $1^{\text {er }}$ tome, $2^{\mathrm{e}}$ édition, Freiburg, Mohr, 1889.

Smith, Barry. Austrian Philosophy. The Legacy of Franz Brentano, Chicago, Open Court, 1994.

Twardowski, Kazimierz. Zur Lehre vom Inhalt und Gegenstand der Vorstellungen. Eine psychologische Untersuchung, Wien, Hölder, 1894 (réimpr. Munich, Philosophia, 1982).

Venn, John. Symbolic Logic, London, MacMillan, 1881. 